\title{
User Interaction Based Recommender System Using Machine Learning
}

\author{
R. Sabitha ${ }^{1}$, S. Vaishnavi ${ }^{2, *}$, S. Karthik ${ }^{1}$ and R. M. Bhavadharini ${ }^{3}$ \\ ${ }^{1}$ Department of Computer Science and Engineering, SNS College of Technology, Coimbatore, 641035, India \\ ${ }^{2}$ Department of Information Technology, Sri Ramakrishna Engineering College, Coimbatore, 641022, India \\ ${ }^{3}$ Department of Computer Science and Engineering, SRM Eswari Engineering College, Chennai, 600089, India \\ ${ }^{*}$ Corresponding Author: S. Vaishnavi. Email: svaishnavi1731@gmail.com \\ Received: 28 March 2021; Accepted: 03 July 2021
}

\begin{abstract}
In the present scenario of electronic commerce (E-Commerce), the indepth knowledge of user interaction with resources has become a significant research concern that impacts more on analytical evaluations of recommender systems. For staying in aggressive E-Commerce, various products and services regarding distinctive requirements must be provided on time. Moreover, because of the large amount of product information available online, Recommender Systems (RS) are required to analyze the availability of consumers, which improves the decision-making of customers with detailed product knowledge and reduces time consumption. With that note, this paper derives a new model called User Interaction based Recommender System (UI-RS) that utilizes the data from multiple sources and opinion-based analysis for sensing the consumer needs and interests. For that, Content-Based Filtering (CBF) analyses various products and determines the likeliness of products based on User Interaction to recommend that to consumers. Then, the product information from multiple sources is combined with DempsterShafer (D-S) evidence theory, and then, decision making for product recommendation is performed with CBF. Moreover, the modified Radial Basis Function Neural Networks (RBFNN) technique has been incorporated for measuring product recommendations. The results show that the proposed model produces better results in providing accurate recommendations to Consumers with a higher rate of coverage and precision, thereby enhancing significant growth in E-Commerce.
\end{abstract}

Keywords: Recommender system; content-based filtering; radial basis function neural networks; user interaction; e-commerce

\section{Introduction}

With the enormous growth of Internet usage for E-Commerce, personalized product recommendation for each consumer has contributed significantly to business society for its improvements. Moreover, it is also to be considered that the number of consumers is significant, and conventional techniques are insufficient for providing personalized recommendations. In today's business scenario, E-Commerce is considered to be associated with user satisfaction, and the success rate of business society is connected to customer loyalty [1]. In recently advanced Internet inducement in many applications, E-Commerce recommender systems

This work is licensed under a Creative Commons Attribution 4.0 International License, which permits unrestricted use, distribution, and reproduction in any medium, provided the original work is properly cited. 
can aid people to live in a better way. In the process of online learning, acquiring an intelligent and personalized RS provide more quality of service than using traditional modes of services [2].

Moreover, the intelligence-based personalized product recommender models have attained great development in present days, according to the enormous growth of the E-Commerce domain and online transactions. RS are Machine Learning (ML) algorithms that filter information from vast volumes of data and provide personalized services to customers. The personalization ideas are provided to users based on items searched and interaction between user and item. The main aim of the RS is to handle information overload by providing relevant E-service. However, based on the enormous amount of user information, developing a product recommender model based on user interests is a tedious process. Fig. 1 explains the typical process of the RS in providing product recommendations.

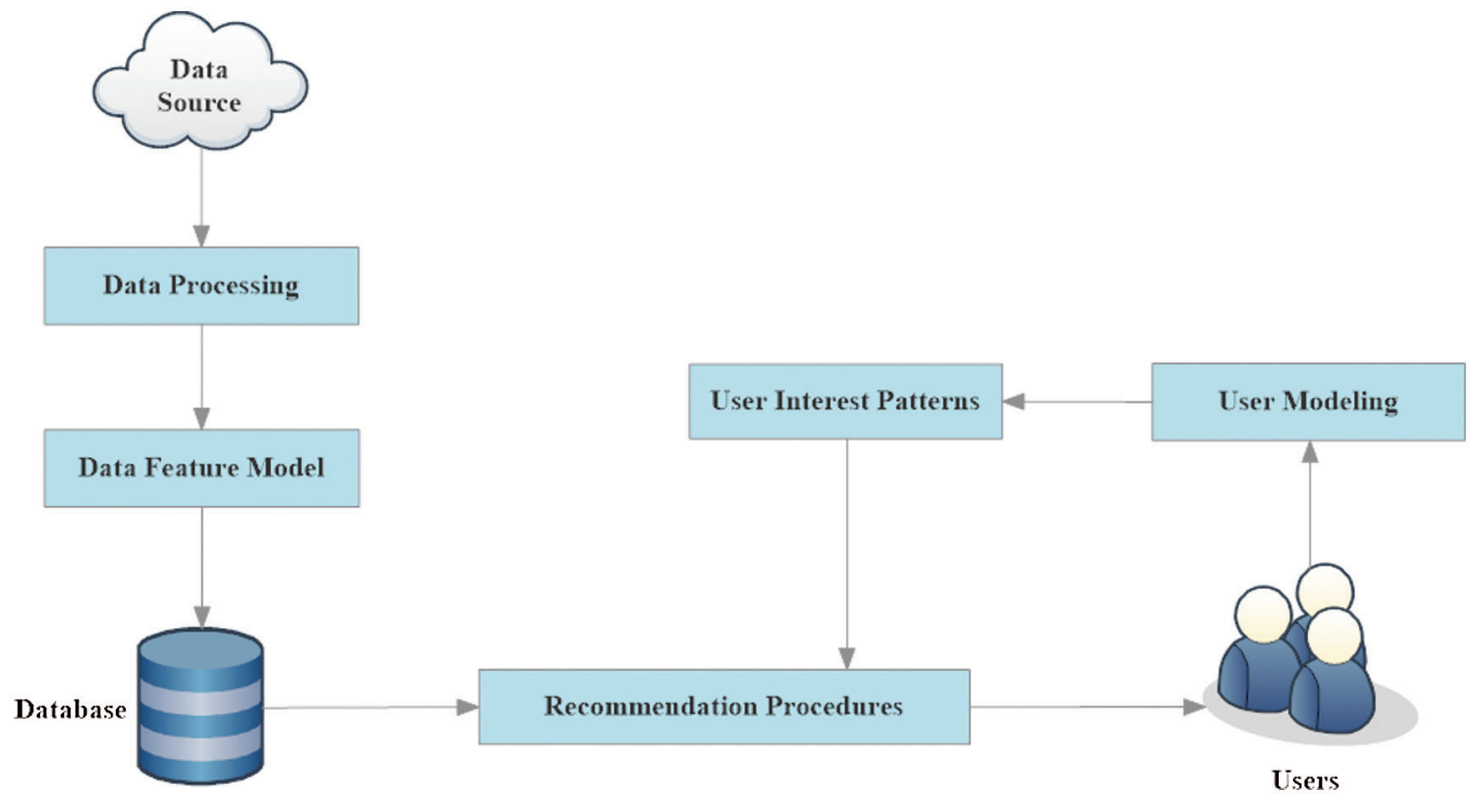

Figure 1: Typical process of recommender model based on user interests

The huge data contains enormous potential for bringing revolt in people's life, specifically in recommender models. Nevertheless, overloading user information is an extraordinary confrontation in using big data. And, the process of extracting relevant or required data from complex user data is still a big challenge. The RS is used to derive the items based on the user's interests from the huge data collection, and the derived results are provided as recommendations for the user in the form of exciting product lists. The usefulness of an item to a user is identified by a function called Utility function, which measures the degree of likeliness of a user towards a product that is frequently characterized by ratings given to a product. The recommender models are generally used in commercial, social networking, and E-Commerce applications such as Amazon, Flipkart, Instagram, Google and Twitter.

For example, an E-Commerce site uses item to item collaborative filtering for providing personalized content to its users. Furthermore, a recommendation can be provided to the users based on the user's previous data through the system. For instance, when a user searches for beauty products online, the RS tends to provide notifications or prompts similar beauty products when the user surfs the web next time. Thus, these systems help individuals to evaluate the alternatives/options based on their interests. The general framework of RS comprises User Interface, Database, Recommending Engine, Database and Results presented in Fig. 2. 


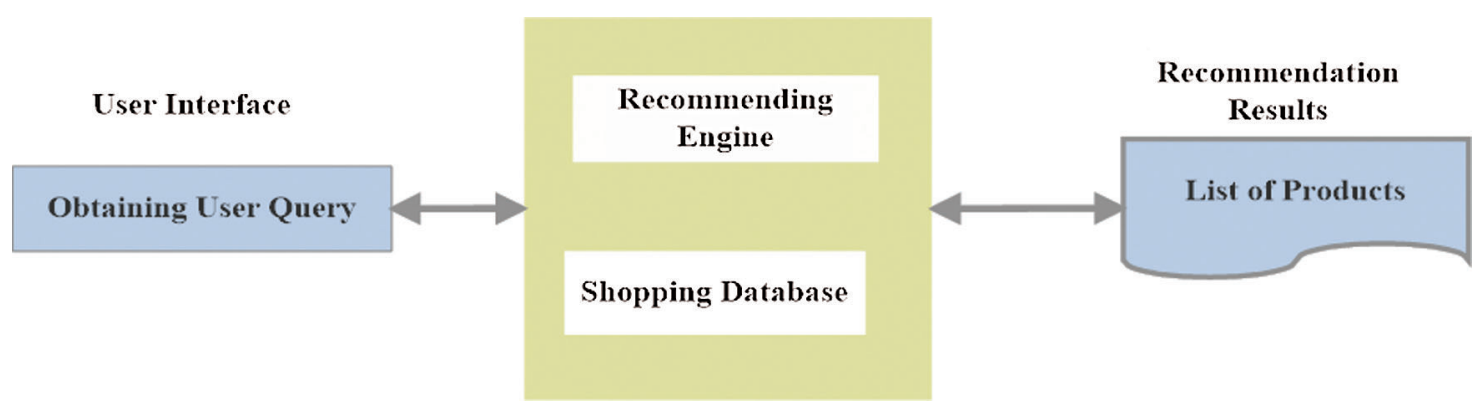

Figure 2: General framework of RS

In conventional methods of RS, the filters that are utilized are given below:

a. Collaborative Filtering:

The Collaborative Filtering model works based on the relationship between products and consumers. Users are clustered into the group with similarities; if a customer shows interest in a product liked by others, then recommendations related to other products, which is shown interest by others in the cluster, will be given in addition to the user. However, the model has some constraints over data sparsity and Cold Start Problem.

\section{b. Content Based Filtering:}

The CBF techniques acquire the products that the consumers have chosen to identify similar products for providing buying suggestions. Since there are huge amounts of heterogeneous data, the model uses user behaviours to provide personalized suggestions.

\section{c. Hybrid Filtering:}

This technique combines the multiple sources of heterogeneous user data to solve the Cold Start problem and data sparsity issues in collaborative filtering. However, because of the heterogeneity of user data, the process may face uneven data distribution and have some complexities and challenges in implementations.

In present days, ML techniques have attained notable results in image processing, voice and language processing and speech and pattern recognition. Moreover, the ML-based models can handle the data heterogeneity. Hence, recommender methods are integrated with ML to cope with the limitations of traditional RS. The proposed model develops a UI-RS for supporting business society in E-Commerce. The contributions of UI-RS are listed as follows,

a) A novel RS based on UI is developed using CBF to analyze user's interest in products.

b) Product information from multiple sources is integrated with the D-S evidence theory.

c) Weight Determination is performed with Modified RBFNN for effectively sorting and listing out the product recommendations.

The rest of this paper is framed as follows: Section 2 discusses the existing RS in E-Commerce. Section 3 clearly describes the computations and procedures for implementing the proposed UI-RS in E-Commerce based business models. The experimental results are provided in Section 4 with some evidence and comparisons for substantiating the proposed model. Lastly, conclusions and future enhancement-based discussions are presented in Section 5. 


\section{Literature Survey}

The E-Commerce based RS is the system to help customer's decision making based on their purchasing behaviour and their product preferences [3]. Moreover, E-Commerce websites' business platforms and product recommendations are broadly used to give users personalized buying services or advertisements in recent electronic markets scenarios. The authors of [4] developed the first RS, and other researchers have developed a wide range of related models for product recommendations based on the data mining process [5] and filtering techniques. In Benbasat et al. [6], a strategic RS has been developed based on CBF techniques. Unlike the traditional techniques that concentrated on some technical recommendation features [7], a few other research works focused on the purchase decisions made by the users or customers [8]. RS confine their usage to E-Commerce and different kinds of needs such as movie recommendations, books, songs, etc., and use different recommendation models to attract and satisfy customer interests [9]. For example, the proposed work discussed the RS for supporting tourism to provide hotel suggestions to the customers before booking [10]. The recommendations were provided based on the opinions and preferences of the customers. Such recommendations face the problems [11] of gathering known ratings, cold start problem, sparsity, scalability and lack of data. Since the model had to be dealt with distinctive user data formats, including numbers, letters, voice, visuals and so on, the RS has been developed in such a manner to support heterogeneous user data [12]. The paperwork in Scholz et al. [13] analyzed that some recent product recommendations could handle multiple data sources, and the customer feedback was used to process the recommendations.

Moreover, the model used multiple feedback types, including voting, ratings, and reviews provided through social media. The RS developed in Xiao et al. [14] intended to achieve relevant suggestions. The relevant recommendations were considered as the recommendations based on the user's choice and likeliness. Furthermore, the paper handled multiple data and customer's opinion-based evaluations for deriving the customer's thoughts and views on a product search through the web. In paper [15], a simple and easy to use recommendation algorithm has been developed, which can be used by non-experts in the field [16].

The quality of product recommendations was considered in Zhang et al. [17]. It was provided in the paper that massive data of consumer reviews and ranks were utilized for giving efficient and quality recommendations since the product suggestion qualities were very much important. Moreover, online purchases and product suggestions have had a great impact on E-commerce. A Recommendation System provides information to find the exact products that the customers searched for and the relevant supporting products. In paper [18], different RS was developed based on different recommendation protocols following the past data consisting of product ratings and reviews. Furthermore, Trust-Aware Recommender System (TARS) has been developed in Hsieh et al. [19] that provided recommendations on shopping, entertainment, sports, etc. And, Knowledge-based Recommender System (KRS) was provided and discussed in [20], which extracted knowledge from users' preferences and product alignment for providing the recommendation lists.

Furthermore, in Burke [21], elaborated survey work has been presented about different RS. The paper explained different RS and the Hybrid Filtering model that combined both the collaborative and CBF techniques. The trends and advantages of a content-based RS have been provided in Burke [22]. The model worked based on collected customer ratings for products and the connectivity between consumers and products for providing the most similar recommendations to the customers. The proposed work in Lops et al. [23] describes using neural networks and genetic algorithms with improved prediction performance. Neural network algorithms like multilayer perceptron, feed-forward network and coactive neuro-fuzzy inference systems were trained and tested with widely used Movielens $100 \mathrm{k}$ and $1 \mathrm{M}$ datasets. Combining genetic algorithms with neural networks improves its performance for the prediction of movies. 


\section{Proposed Model}

The proposed UI-RS uses multiple sources to observe customer requirements and opinions. Moreover, CBF is used for computing the likeliness of products based on weights. The data from multiple sources are integrated using D-S evidence theory. Further, the final listing of product recommendations is processed with modified RBFNN for supporting E-Commerce effectively. The proposed work contains the following sub-sections.

a) Acquiring Multiple-Data Sources

b) CBF based Weight Computation

c) Decision Integration with D-S Evidence Theory

d) Modified RBFNN for Final Results.

\subsection{Acquiring Multiple-Data Sources}

The RS requires accurate and integrated user data in the E-Commerce-based business that comprises the customer features and requirements. Here, the proposed RS model uses microformats for customer data representation, fusion, storage and administration. The foreign commercial sites have used micro-formats for data integration and achieved greatly in YouTube, Amazon, etc. The user data such as Gender, Name, Age and Locations are needed by the RS required for shopping zones. However, the inclusion of data such as potential requirements of customers, immediate product requirements, user's recent interests, etc., are still to be done for framing efficient product recommendations. This paper proposes that the RS can acquire user data based on applications and usage.

Moreover, for providing timely product recommendations, the data sharing process can be used. The RS can obtain the personal behaviours and customer's recent interests using their social platform activities and mobile numbers. Additionally, customers' purchase history is also obtained from the databases of their shopping platforms from the mobile number of customers. Hence, the model acquires two categories of user data based on the UI with the shopping platforms, and the process of data acquisition is presented in Fig. 3.

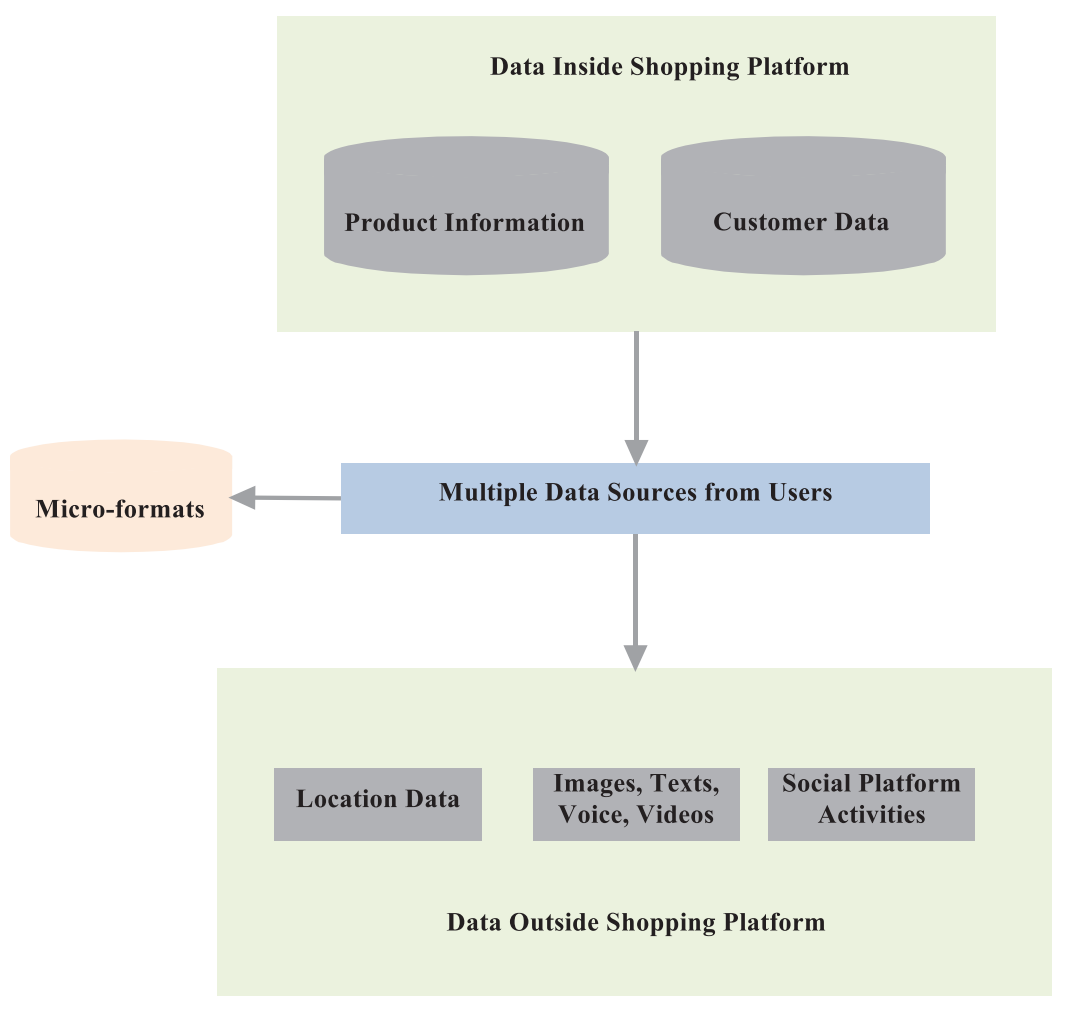

Figure 3: Acquiring multiple-data sources of users 


\subsection{CBF Based Product Similarity Computation}

Here, $\mathrm{CBF}$ evaluates products and performs similarity identifications with the active customers to provide product recommendations. The model acquires the user interactions according to the data retrieval and analysis and the user's purchase history from the databases of shopping platforms. The data can be described with the classes that are provided with weights. Computing similarities between items or users calculate these weights. It is significant to compute the similarity as it is essential to find items or users similar to a users' interest. Based on the classes and the customer preferences, the products are recommended to the active users, and a set of features are derived from that. The features are evaluated based on keywords that denote recommendations. Further, weights are computed based on the occurrence rate of keywords called Term Frequency (TF). And, the TF is calculated as follows, Eq. (1)

$\mathrm{TF}_{\mathrm{a}, \mathrm{b}}=\frac{\mathrm{f}_{\mathrm{a}, \mathrm{b}}}{\max _{\mathrm{m}, \mathrm{f}, \mathrm{b}}}$

where, ' $\mathrm{f}_{\mathrm{a}, \mathrm{b}}$ ' is the number of occurrences of keywords ' $\mathrm{k}_{\mathrm{a}}$ ' that are presented in the document $\mathrm{d}_{\mathrm{b}}$ and the maximum frequency is given as ' $\mathrm{f}_{\mathrm{m}, \mathrm{b}}$ '. ' $\mathrm{f}_{\mathrm{m}, \mathrm{b}}$ ' is the number of occurrences of all keywords ' $\mathrm{k}_{\mathrm{z}}$ ' that are presented in. ' $d_{b}$ '. Further, the Inverse Document Frequency (IDF) is calculated as Eq. (2)

$\mathrm{IDF}_{\mathrm{a}}=\log \frac{\mathrm{N}}{\mathrm{n}_{\mathrm{a}}}$

Then, the weight of each keyword for providing product recommendation is computed as, Eq. (3)

$\mathrm{W}_{\mathrm{a}, \mathrm{b}}=\mathrm{TF}_{\mathrm{a}, \mathrm{b}} \times \mathrm{IDF}_{\mathrm{a}}$

Similarity measurement is related to the distance between the items. Here, TF-IDF is used to compute weight, which focuses on feature extraction. Using this weight, similarity measurement is done. Finally, search keywords are gathered, based on which similarity with an unseen item has been computed. Based on the results, a content-oriented profile has been generated for customers based on their product likeliness, product ratings, keywords and other features. The work process of the proposed RS is presented in Fig. 4.

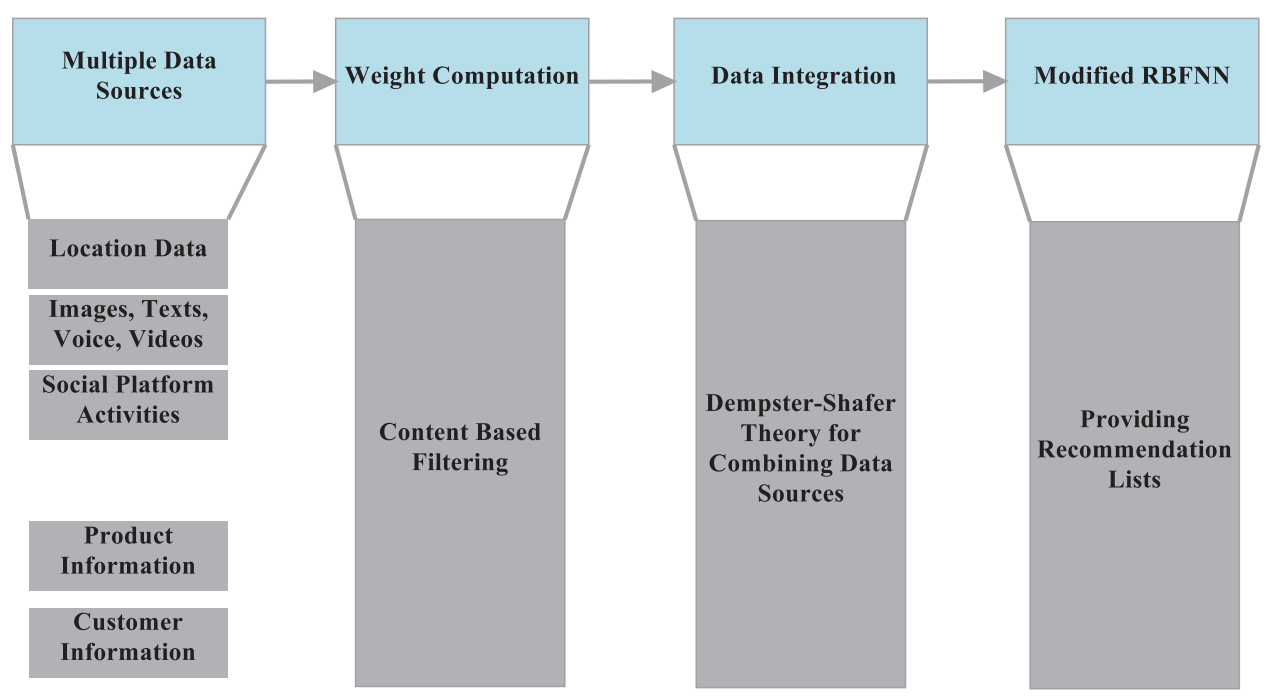

Figure 4: Work flow of proposed RS 


\subsection{Data Integration with Dempster-Shafer (D-S) Theory}

This evidence theory is developed in such a manner to support Reasoning under Uncertainty (RUU). The fusion rules of evidence $\left\{\mathrm{e}_{1}, \mathrm{e}_{2}, \ldots, \mathrm{e}_{\mathrm{n}}\right\}$ are provided in the following Eq. (4) as,

$\mathrm{e}(\mathrm{A})= \begin{cases}0, & \mathrm{~A}=\varphi \\ (1-\mathrm{K})^{-1} \sum_{\cap \mathrm{A}_{\mathrm{i}}=\mathrm{A}} \prod_{\mathrm{i} \leq \mathrm{j} \leq \mathrm{n}} \mathrm{e}_{\mathrm{j}}\left(\mathrm{A}_{\mathrm{i}}\right) & \mathrm{A} \neq \varphi\end{cases}$

From that, the probability assignment persuades $\sum_{i} e_{j}\left(A_{i}\right)=1$ and $K=\sum_{\cap A_{i}=\varphi} \sum_{i} e_{j}\left(A_{i}\right)$ denotes divergences between the evidence. Moreover, for efficient data integration, reliability-based modifications and local divergence allocations have been done. And, the modifications in general D-S theory in the proposed work is presented below.

i) Reliability-based modifications in evidence structure are performed. The evidence authority is given as $\alpha_{\mathrm{i}}\left(\alpha_{\mathrm{i}}-1\right)$ denotes the relative accountability between the evidence. For compelling performances, the highest rate of accountability is set as 1 , where $\alpha_{\mathrm{i}}$ is considered as the correction parameter that changes the base evidence structure $E_{0}$. Then, the additional probability is constructed for the anonymous condition $\mathrm{e}(\mathrm{Q})$. The value of the probability assignment of the changed evidence body (E) is derived from the following Eq. (5).

$\mathrm{E}=\left[\mathrm{e}\left(\mathrm{A}_{1}\right) \mathrm{e}\left(\mathrm{A}_{2}\right) \ldots \mathrm{e}\left(\mathrm{A}_{\mathrm{n}}\right) \mathrm{e}(\mathrm{Q})\right]$

ii) Local Divergence Allocation for integration rule framing is performed next. The division between two focal ends that causes the divergences is done as given below Eq. (6)

$$
\left\{\begin{array}{l}
e_{j K}(A)=\sum_{Y \cap Z=X} e_{j}(Y) e_{k}(Z)+f(X) \\
f(X)=\sum_{X \cap D=\varphi}\left(\frac{e_{j}^{2}(X) e_{k}(D)}{e_{j}(X)+e_{k}(D)}+\frac{e_{K}^{2}(X) e_{j}(D)}{e_{j}(D)+e_{k}(A)}\right)
\end{array}\right.
$$

Here, $\mathrm{B}, \mathrm{C}, \mathrm{D} \subset\left\{\mathrm{A}_{1}, \mathrm{~A}_{2}, \ldots, \mathrm{A}_{\mathrm{Q}}\right\}$ and a new evidence structure. ' $\mathrm{e}_{\mathrm{jK}}(\mathrm{A})$ ' are derived and given for normalization. Moreover, in this work, the product information, customer information, location, past customer purchases, and other activities are considered evidence, and statistical evaluations are processed to define their reliability.

\subsection{Modified RBFNN for Generating Product Recommendations}

Modified RBF Neural Networks are used to generate the product recommendation lists based on the previous data integration results in the proposed model. The network structure comprises three layers: Input, Middle, and Output layer, as presented in Fig. 5.

RBFNN neural networks are preferred over other neural networks due to their rapid learning capability. This neural network covers three layers, where the network architecture's input layer is considered, the linear layer containing the data sources for gathering user interactions. The user's evidence integrated into the previous step is given as input for the neural network. The next hidden or Gaussian Layer is considered the middle layer consisting of activation units implemented as Gaussian functions. The Radial Basis function assigns an absolute real value for each input, measuring distance. The output layer is the Softmax layer to produce the list of product recommendations as output. In the middle layer, Gaussfunction is framed as presented in the following Eq. (7). 


$$
\mathrm{b}_{\mathrm{i}}=\exp \left(-\frac{\left\|\mathrm{a}-\mathrm{z}_{\mathrm{i}}\right\|^{2}}{\sigma_{\mathrm{i}}^{2}(\mathrm{a})}\right)
$$

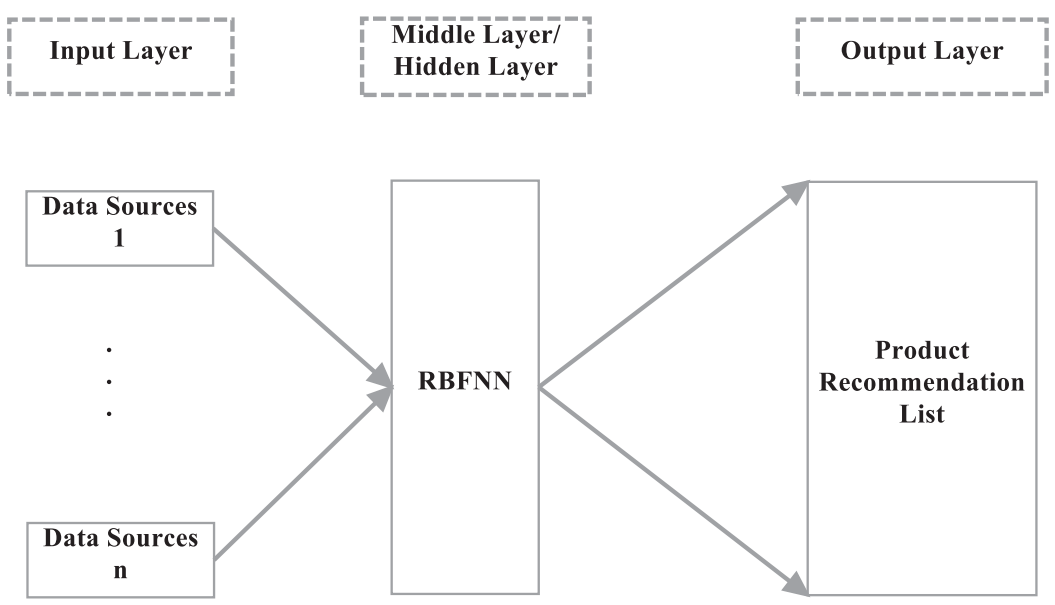

Figure 5: RBFNN architecture in UI-RS

Here, ' $z$ ' ' is considered as the central position, and ' $\sigma$ ' represents the variance value obtained from input sources. Furthermore, the output layer utilizes the transfer function as given in (8), where ' $z$ ' is the number of classified product recommendations and ' $b_{i}$ ' is the neuron output, Eq. (8)

$$
b_{i}=\frac{\exp \left(a_{i}\right)}{\sum_{i=1}^{z} \exp \left(a_{i}\right)}
$$

For providing appropriate or relevant product recommendations to the users, effective decision making is processed in this section. Since the multiple sources of users are variant and have different impacts, those play a vital role. Hence, the Rating of different data sources varies concerning the purpose of RS. Here, the trained and modified RBFNN is used to provide the product recommendations to the users with respect to the previous computations. Finally, the output is given as, Eq. (9)

$\mathrm{B}^{\text {tot }}=\left\{\mathrm{b}_{1}^{\text {tot }}, \mathrm{b}_{2}^{\text {tot }}, \ldots, \mathrm{b}_{\text {sum }}^{\text {tot }}\right\}$

Here, 'sum' represents the total number of data sources based on the UI with the online shopping websites and ' $B^{\text {tot }}$ ' is the weight for product recommendations that are computed from the defined architecture and the integrated sources. Furthermore, the factor ' $b_{i}^{\text {tot }}$ ' is the measure of relative support of data between multiple sources. Based on this deep learning concept, the highest weight rates of sources are given as product recommendations. With ' $B^{\text {tot }}$ ', weight rates of sources, i.e., user interactions details, have been found. RBFNN gives an absolute real distance between the sources, which helps to identify the similarities and weight computation. On finding the similarity weight, users' interests are discovered, which aids in suggesting product recommendations.

\subsection{Modified RBFNN for Generating Product Recommendations}

The performance of the proposed UI-RS is performed based on the accuracy rates of product recommendations that are very much productive and profitable for the E-Commerce environment. Here, the factors such as Result Precision, Recall, Error Rate, Rate of Coverage (RoC) and Processing Speed in presenting recommendations are considered for model analysis. Moreover, the evaluations are carried out based on the acceptance ratio of the targeted customers. Finally, the performance analysis is done to show 
that the proposed work provides practical product recommendations. For example, the Result Precision is calculated based on the following Eq. (10)

Result Precision $(\mathrm{RP})=\frac{\mathrm{X}}{\mathrm{Y}+\mathrm{X}} \times 100 \%$

With respect to the multiple combined sources, ' $\mathrm{X}$ ' denotes the number of likeliness obtained for a particular product, and ' $\mathrm{Y}$ ' denotes the number of products that the users do not like. And, ' $Y+X$ ' derives the total amount of product recommended by UI-RS to a single customer. Hence, the recall result can be calculated by good product recommendations to the model's total number of product recommendations. Further, the recall rate is computed as in Eq. (11).

Recall Rate $(R R)=\frac{X}{Z+X} \times 100 \%$

' $Z$ ' denotes the number of effective products but not provided on the product recommendation lists. Further, F-Measure is derived from RP and RR as given in Eq. (12).

F-Measure $=2 \times \frac{\mathrm{RP} \times \mathrm{RR}}{\mathrm{RP}+\mathrm{RR}}$

Additionally, Mean Absolute Error (MAE) for computing the exact rate of error between the predicted and the actual rates for measuring the accuracy rate of recommendations and the equation for MAE is computed as, Eq. (13)

MAE $=\frac{\sum_{\mathrm{i}=1}^{\mathrm{N}}\left(\mathrm{x}_{\mathrm{i}}-\mathrm{y}_{\mathrm{i}}\right)}{\mathrm{N}}$

' $x_{i}$ ' is the predicted rate and ' $y_{i}$ ' is the actual rate. And, Rate of Coverage (RoC) is calculated as, Eq. (14)

$\operatorname{RoC}(\mathrm{t})=\sum(\mathrm{RR}(\mathrm{t}) \cap \mathrm{RS}(\mathrm{t})) / \sum \mathrm{RR}(\mathrm{t})$

' $R R(t)$ ' is the set of products recommended, and ' $R S(t)$ ' denotes the list of selected resources by the UI$\mathrm{RS}$ at an instant ' $\mathrm{t}$ '. For an efficient RS, the RoC must be higher for satisfying the user requirements.

On applying the user interaction model to the data, user interactions are gathered in various formats from multiple data sources. Collected user and data items are analyzed for computing similarity. The parameter "result precision" considered in the model identifies how close the data points are. This has been achieved by computing the similarities between data items. Ratings have been used to identify the likeliness of a product by a user. Recall rate is used to identify the item or document relevant to the query/request of the customers. These relevant items are gathered by providing input sources to RBFNN, and the hidden layer employing the Gaussian function provides the list of product recommendations.

Furthermore, the accuracy of the recommendations is measured by F-measure by combining the result precision and recall rate calculated earlier. In terms of the error rate, the difference between the observed and true values yields the mean absolute error, which is used to calculate the error rate of the UI model. Finally, $\mathrm{RoC}$ is calculated to ensure the coverage of items for recommendation as comprehensive product coverage contributes to correct recommendations. RoC shall be determined by combining a list of suggested products with a set of products drawn from different resources at a given time.

\section{Results and Discussions}

For experimentations, the raw data from the online shopping site www.askboutique.com is considered, and the data of 120 customer samples are chosen for the proposed model to process. Each sample comprises 
the customer's age, gender, product feedback, and purchase history. With respect to products of the sites, product quality-based reviews, ratings and discounts are mainly considered for presenting that for recommendations. Based on the input sources from sites, the operations are carried out in UI-RS, depending on the user interaction with the shopping site. Moreover, the model is evaluated with respect to the factors presented in Section 3.5. Finally, the results are compared with the existing models such as TARS and KRS.

In Figs. 6 and 7, the precision and recall rates of proposed UI-RS for providing product recommendations are evaluated and presented. In Fig. 6, it is observed that the proposed model obtained a higher rate of precision than other models in producing similar product recommendations to the users based on their interactions and interests over the online purchases. Based on user interactions with the online shopping site, relevant recommendations are provided to the customers. The recall rate results are presented in Fig. 7, as mentioned earlier, which is also greater among other works for the exact recommendation for users. Moreover, it is measured against the number of users in the considered shopping sites.

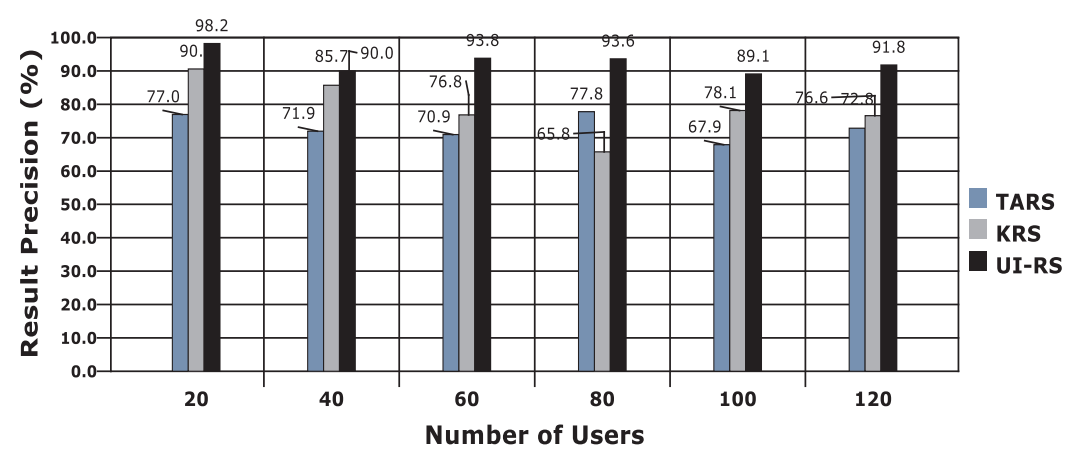

Figure 6: Comparison of precision models

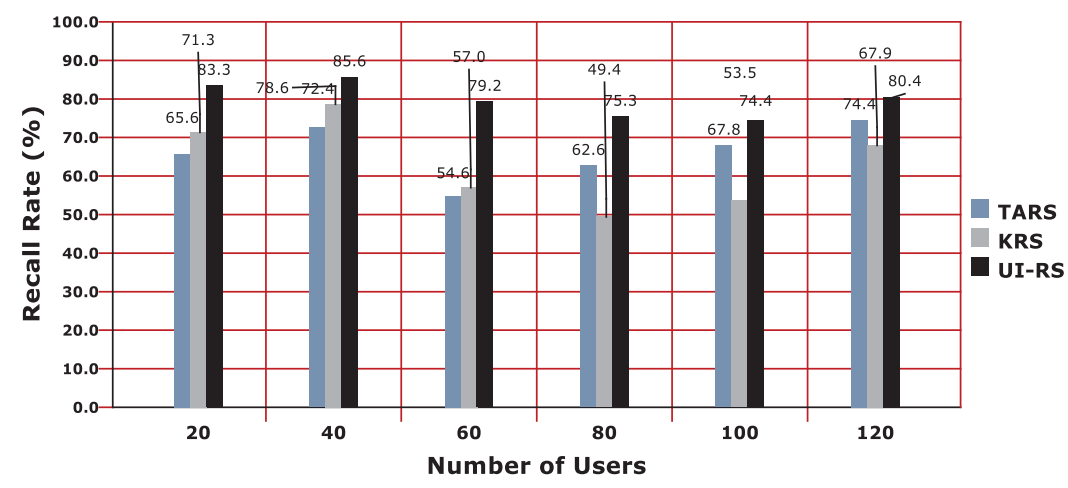

Figure 7: Recall rate vs. Number of users

For an efficient RS, the error rate in providing product recommendations must be lesser than other works, and the results are plotted in Fig. 8 with respect to the number of users. The obtained error rate in the proposed model is about 0.00755 (on average), which is comparatively lower than the other works.

Fig. 9 displays that with the seamless enhancement of customer data in the RS, the coverage rates raise continuously till the targeted customer numbers are reached. Moreover, the proposed model uses effective techniques and optimization methods, thereby providing the highest coverage rate, computed as in 
Bobadilla et al. [24]. The highest RoC results in a great change in the customer number utilizing the particular business domain with respect to product promotions, advertisements and other deeds [25].

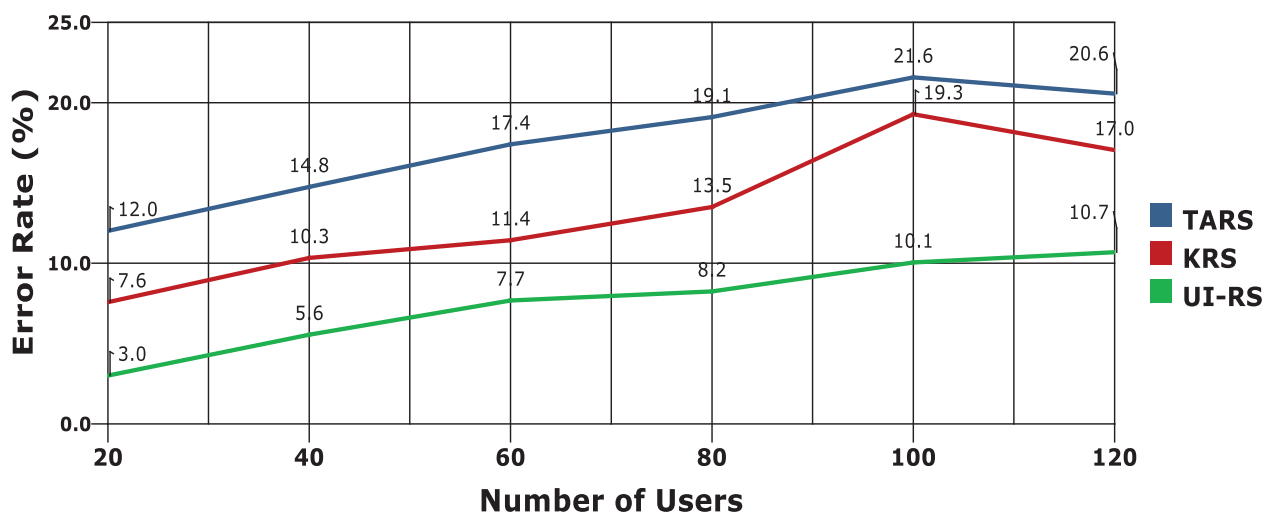

Figure 8: Analysis of error rate

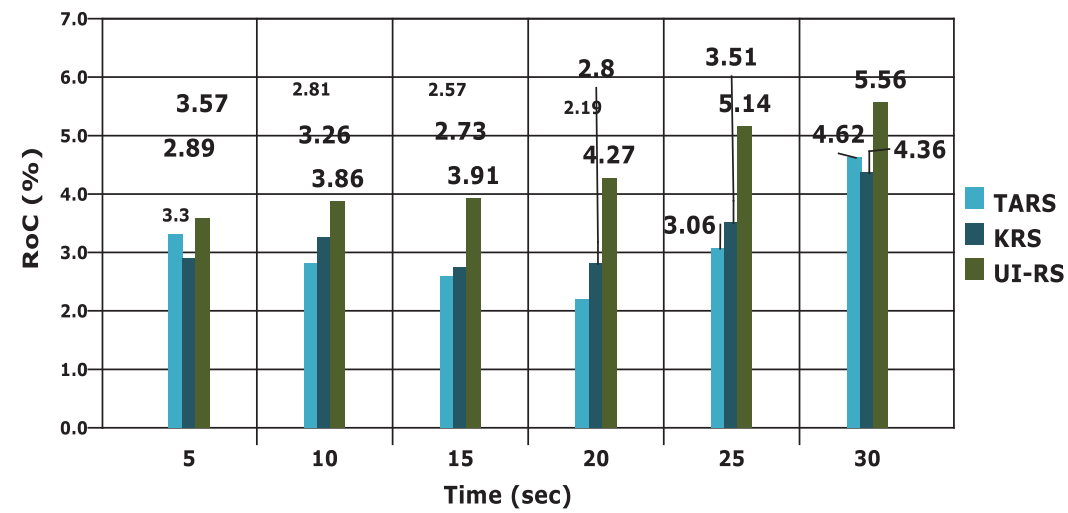

Figure 9: RoC of users with time

The processing speed of RS in providing product recommendations is a significant factor to be considered in performance analysis. In standard RS, the transaction database is taken into account for recommendations, and wherein the proposed model uses the multiple source acquisition that speeds up the process considerably [26]. The values obtained for processing time measurement in the traditional and proposed model are presented in Tab. 1. And, Fig. 10 presents the values plotted against the number of users on the online shopping website. Observing the graph shows that the proposed RS takes minimal time for providing recommendations to the customers, which increases the sales rate of the site in an effective manner [27-29].

Table 1: Values for processing time analysis by recommending products

\begin{tabular}{lllllll}
\hline Models & 20 & 40 & 60 & 80 & 100 & 120 \\
\hline TARS & 0.213 & 0.223 & 0.223 & 0.222 & 0.275 & 0.377 \\
KRS & 0.09 & 0.182 & 0.251 & 0.252 & 0.368 & 0.425 \\
UI-RS & 0.069 & 0.087 & 0.15 & 0.155 & 0.201 & 0.249 \\
\hline
\end{tabular}




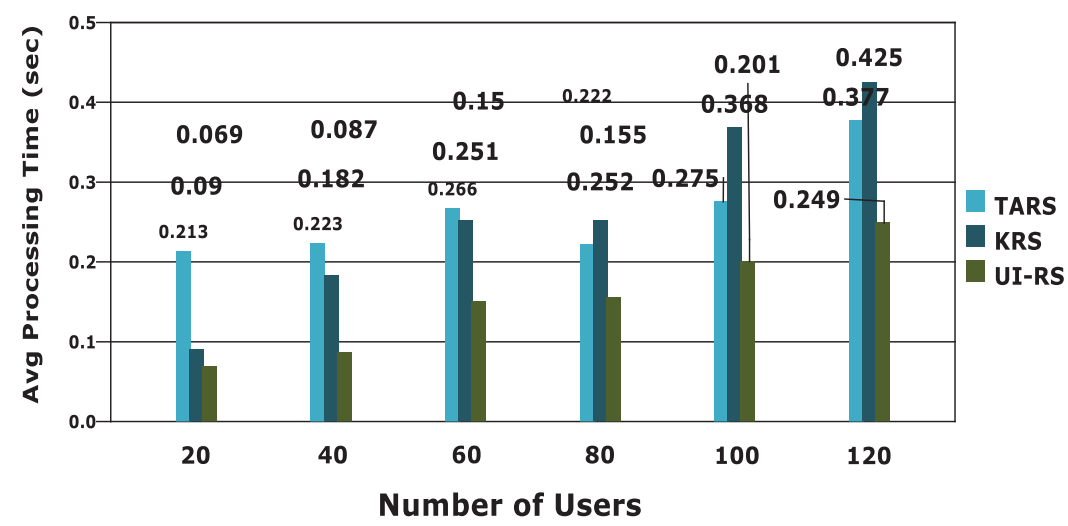

Figure 10: Processing time evaluations and comparisons

\section{Conclusions and Future Work}

In this paper, a novel User Interaction based Recommendation System (UI-RS) is proposed for handling multiple user sources such as the location of data, product and customer information, social platform activities, images, videos, voices and texts for enhancing the accuracy in generating product recommendations with minimal processing speed. Moreover, the proposed model utilizes CBF to determine customers' likeliness over the products of a particular business website. And further, D-S Evidence theory is enforced for data integration and RBFNN is used for processing the inputs and presenting the lists of product recommendations based on their computed weights. The result evaluations show that the proposed RS results provide good product suggestions to the customers with more precision in minimal time. And, the results also show that UI-RS generates the highest RoC than other traditional models. In future, the work can be extended in such a manner to provide recommendations for particular products by considering advanced options such as navigational actions of users, cookies data and so on.

Funding Statement: The authors received no specific funding for this study.

Conflicts of Interest: The authors declare that they have no conflicts of interest to report regarding the present study.

\section{References}

[1] J. Davidson, B. Liebald, J. Liu, P. Nandy, T. V. Vleet et al., "The you tube video recommendation system," in Proc. of ACM, New York, pp. 293-296, 2010.

[2] W. D. Mcdonald and S. M. Ackerman, "Expertise recommender: A flexible recommendation system and architecture," in Proc. of the 2000 ACM Conf. on Computer Supported Cooperative Work, New York, pp. 231-240, 2000.

[3] Y. Guo, M. X. Wang and X. Li, "An interactive personalized recommendation system using the hybrid algorithm model," Symmetry, vol. 9, no. 10, pp. 216-224, 2017.

[4] I. Yuto, U. Takahiro and T. Ichi, "Design and evaluation of a movie recommendation system showing a review for evoking interested," International Journal of Web Information Systems, vol. 13, no. 2, pp. 72- 84, 2017.

[5] G. Shani and A. Gunawardana, "Evaluating recommendation systems," in Recommender Systems Handbook. Berlin, Germany: Springer, pp. 257-297, 2011.

[6] I. Benbasat and W. Wang, "Trust in and adoption of online recommendation agents," Journal of the Association for Information Systems, vol. 6, no. 3, pp. 72-101, 2005.

[7] D. Goldberg, D. Nichols, B. M. Oki and D. Terry, "Using collaborative filtering to weave an information tapestry," Communications of the ACM, vol. 35, no. 12, pp. 61-70, 1992. 
[8] M. Masoud and S. Mehdi, "Improving recommender system's performance on cold-start users and controversial items by a new similarity model," International Journal of Web Information Systems, vol. 12, no. 2, pp. 126-149, 2016.

[9] M. Buvana, K. Muthumayil and T. Jayasankar, "Content-based image retrieval based on hybrid feature extraction and feature selection technique pigeon inspired based optimization," Annals of the Romanian Society for Cell Biology, vol. 25, no. 1, pp. 424-443, 2021.

[10] K. W. Cheung, J. T. Kwok, M. H. Law and K. C. Tsui, "Mining customer product ratings for personalized marketing," Decision Support Systems, vol. 35, no. 2, pp. 231-243, 2003.

[11] Z. J. Lin, "An empirical investigation of user and system recommendations in e-commerce," Decision Support Systems, vol. 68, no. 3, pp. 111-124, 2014.

[12] L. C. Cheng and M. J. Jhang, "A novel approach to exploring maximum consensus graphs from users' preference data in a new age environment," Electronic Commerce Research, vol. 15, no. 4, pp. 543-569, 2015.

[13] M. Scholz, V. Dorner, M. Franz and O. Hinz, "Measuring consumer's willingness to pay with utility-based recommendation systems," Decision Support Systems, vol. 72, no. 2, pp. 60-71, 2015.

[14] B. Xiao and I. Benbasat, "E-Commerce product recommendation agents: Use, characteristics, and impact," Management Information Systems Quarterly, vol. 31, no. 1, pp. 137-209, 2007.

[15] H. Liu, J. He, T. Wang, W. Song and X. Du, "Combining user preferences and user opinions for accurate recommendation," Electronic Commerce Research and Applications, vol. 12, no. 1, pp. 14-23, 2013.

[16] M. Ibrahim and I. Bajwa, "Design and application of a multi-variant expert system using apache hadoop framework," Sustainability, vol. 10, no. 11, pp. 4280-4293, 2018.

[17] J. J. Zhang and Z. Mao, "Image of all hotel scales on travel blogs: Its impact on customer loyalty," Journal of Hospitality Marketing \& Management, vol. 21, no. 2, pp. 113-131, 2012.

[18] Y. Koren, R. Bell and C. Volinsky, "Matrix factorization techniques for recommender systems," Computer IEEE Computer Society, vol. 42, no. 8, pp. 30-37, 2009.

[19] M. Y. Hsieh, W. K. Chou and K. C. Li, "Building a mobile movie recommendation service by user rating and APP usage with linked data on Hadoop," Multimedia Tools and Applications, vol. 76, no. 3, pp. 3383-3401, 2017.

[20] M. Paolo and A. Paolo, "Trust-aware recommender systems," in Proc. of RecSys'07: ACM Conf. on Recommender Systems, Madison, pp. 17-24, 2007.

[21] R. Burke, "Knowledge-based recommender systems," Encyclopedia of Library and Information Systems, Supplement, vol. 32, pp. 175-186, 2000.

[22] R. Burke, "Hybrid recommender systems: Survey and experiments," User Modeling and User-Adapted Interaction, vol. 12, no. 4, pp. 331-370, 2002.

[23] P. Lops, M. D. Gemmis and G. Semeraro, "Content-based recommender systems: State-of-the-art and trends," in Recommender Systems Handbook. Boston, MA, USA: Springer, pp. 73-105, 2011.

[24] J. Bobadilla, F. Ortega, A. Hernando and A. Gutiérrez, "Recommender systems survey," Knowledge-Based Systems, vol. 46, no. 4, pp. 109-132, 2013.

[25] G. Adomavicius and A. Tuzhilin, "Toward the next generation of recommender systems: A survey of the stateof-the-art and possible extensions," IEEE Transactions on Knowledge and Data Engineering, vol. 17, no. 6, pp. 734-749, 2005.

[26] S. Berna, B. Erkan and G. Mehmet Serdar, "Evolutionary neural networks for improving the prediction performance of recommender systems," Turkish Journal of Electrical Engineering \& Computer Sciences, vol. 29, no. 1, pp. 62-77, 2021.

[27] M. Nitin, "Research problems in recommender systems," in Proc. J. Phys.: Conf. Ser. 1717 012002, 2021.

[28] G. Namita, C. Prasenjit and C. Tanupriya, Smart and sustainable intelligent systems. Sustainable Computing and Optimization, John Wiley \& Sons, pp. 173-180, 2021. [Online]. Available: https://onlinelibrary.wiley.com/doi/ book/10.1002/9781119752134.

[29] Kim Falk, Practical Recommender Systems. Shelter Island: Manning Publications Co., 2019. 